\title{
Coherent Stranski-Krastanov growth in 1+1 dimensions with anharmonic interactions: An equilibrium study
}

\author{
Elka Korutcheva* \\ Departamento de Fisica Fundamental, Universidad Nacional de Educacion a Distancia, 28040 Madrid, Spain \\ Antonio M. Turiel \\ Laboratoire de Physique Statistique, Ecole Normal Superieure, 24, rue Lhomond, 75231 Paris Cedex-05, France \\ Ivan Markov ${ }^{\dagger}$ \\ Institute of Physical Chemistry, Bulgarian Academy of Sciences, 1113 Sofia, Bulgaria
}

(Received 13 January 2000)

\begin{abstract}
The formation of coherently strained three-dimensional (3D) islands on top of the wetting layer in the Stranski-Krastanov mode of growth is considered in a model in $1+1$ dimensions accounting for the anharmonicity and nonconvexity of the real interatomic forces. It is shown that coherent 3D islands can be expected to form in compressed rather than expanded overlayers beyond a critical lattice misfit. In expanded overlayers the classical Stranski-Krastanov growth is expected to occur because the misfit dislocations can become energetically favored at smaller island sizes. The thermodynamic reason for coherent 3D islanding is incomplete wetting owing to the weaker adhesion of the edge atoms. Monolayer height islands with a critical size appear as necessary precursors of the 3D islands. This explains the experimentally observed narrow size distribution of the 3D islands. The 2D-3D transformation takes place by consecutive rearrangements of monoto bilayer, bi- to trilayer islands, etc., after the corresponding critical sizes have been exceeded. The rearrangements are initiated by nucleation events, each one needing to overcome a lower energetic barrier than the one before. The model is in good qualitative agreement with available experimental observations.
\end{abstract}

\section{INTRODUCTION}

The preparation of arrays of defect-free three-dimensional (3D) nanoscale islands has been a subject of intense research in the last decade owing to possible optoelectronic applications as quantum dots. The latter are promising for fabrication of lasers and light emitting diodes. ${ }^{1-4}$ Recently, the instability of two-dimensional (2D) growth against the formation of coherently strained 3D islands in highly mismatched heteroepitaxial systems has been successfully used to produce quantum dots. This is the well known StranskiKrastanov (SK) growth mode where the decrease of the strain energy in the 3D islands overcompensates the contribution of the surface energy.

When the adhesion forces between the substrate and film materials overcompensate the strain energy stored in the overlayer owing to the lattice mismatch, a thin pseudomorphous wetting layer consisting of an integer number of monolayers is first formed by a layer-by-layer mode of growth. This kind of growth cannot continue indefinitely because of the accumulation of strain energy and the disappearance of the energetic influence of the substrate after several atomic diameters. Then, in the thermodynamic limit, unstrained 3D islands are formed and grow on top of the wetting layer, the lattice misfit being accommodated by misfit dislocations (MDs) at the wetting layer-the 3D island boundary. ${ }^{5,6}$ Thus the wetting layer and the 3D islands represent different phases in the sense of Gibbs, ${ }^{7}$ separated by an interphase boundary. The energy of the boundary is given by the energy of the array of MDs. This is the classical Stranski-Krastanov mechanism of growth ${ }^{8}$ [see Fig. 1(a)].
However, it has been found that under certain conditions coherently strained (dislocation-free) 3D islands are formed on top of the wetting layer [Fig. 1(b)]. These islands are strained to fit the wetting layer in the middle of their bases but are more or less strain-free near their top and side walls. ${ }^{9,10}$ Such coherently strained islands are formed at large positive misfits when the lattice parameter of the overlayer is larger than that of the substrate and the overlayer is compressed. It has also been observed that the size distribution of the 3D islands is very narrow. The above observations have been reported for the growth of $\mathrm{Ge}$ on $\mathrm{Si}(100),{ }^{2,4,11-16}$ InAs on $\mathrm{GaAs}(100),{ }^{17-22} \mathrm{InGaAs}$ on $\mathrm{GaAs},{ }^{3,23-25}$ and $\mathrm{InP}$ on $\mathrm{In}_{0.5} \mathrm{Ga}_{0.5} \mathrm{P} .{ }^{26}$ In all cases the lattice misfit is positive and very large $(4.2,7.2$, and $\approx 3.8 \%$ for $\mathrm{Ge} / \mathrm{Si}$, InAs/GaAs, and

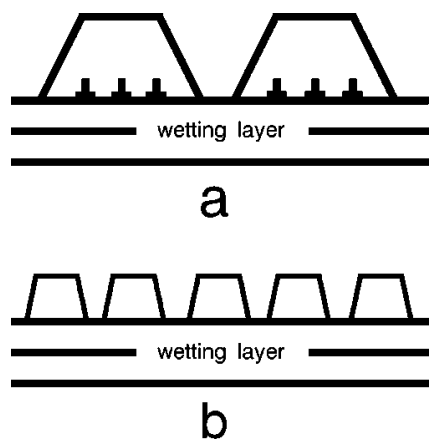

FIG. 1. Schematic representation of (a) classical SK growth, and (b) coherent SK growth. In the latter case the sidewalls are shown steeper to demonstrate the compression exerted by the wetting layer. The MDs in (a) are denoted by inverse T's. 
InP/ $/ \mathrm{In}_{0.5} \mathrm{Ga}_{0.5} \mathrm{P}$, respectively) for semiconductor materials, which are characterized by directional and brittle chemical bonds. The only exception to the authors' knowledge is the system $\mathrm{PbSe} / \mathrm{PbTe}(111)$ in which the misfit is negative $(-5.5 \%)$ and the overlayer is expanded. ${ }^{27}$ However, the authors of Ref. 27 note that, whereas the in-plane lattice parameter of the $\mathrm{PbSe}$ wetting layer is strained to fit the $\mathrm{PbTe}$ substrate exactly, the parameter of the 3D islands rapidly decreases, reaching $95 \%$ of the bulk PbSe lattice constant at about 4 monolayers coverage. ${ }^{27}$ One could speculate that the lattice misfit is accommodated by MDs introduced at the onset of the 3D islanding.

Whereas the classical SK growth is more or less clear from both thermodynamic and kinetic points of view, the formation of coherent 3D islands still lacks satisfactory explanation. We can consider as a first approximation the formation of coherent 3D islands in SK growth as homoepitaxial growth on a uniformly strained crystal surface, both film and substrate materials having one and the same bonding. In this case, it is not clear what is the thermodynamic driving force for 3D islanding if the islands are coherently strained to the same degree as the wetting layer. It is also not clear why coherent 3D islands are observed in compressed rather than in expanded overlayers. Another question that should be answered is why the formation of coherent 3D islands requires a very large value of the positive misfit. The reason for the narrow size distribution is still unclear although much effort has been made to elucidate the problem. ${ }^{28,29}$ Finally, the mechanism of formation of coherent $3 \mathrm{D}$ islands is still an open question.

Two major approximations are usually made when dealing theoretically with the formation of coherently strained 3D islands. The first is the use of the linear theory of elasticity in order to compute the strain contribution to the total energy of the islands. $9,10,28,30-40$ However, the validity of the latter is hard to accept, bearing in mind the high values of the lattice mismatch. As will be shown below, the MDs differ drastically in compressed and expanded films. Second, it is commonly accepted that the interfacial energy between the wetting layer and the dislocation-free 3D islands is sufficiently small that it can be neglected in the case of coherent SK growth. This is equivalent to the assumption that the substrate (the wetting layer) wets the 3D islands completely. ${ }^{28,31-33,35-39}$ In fact this assumption rules out 3D islanding from a thermodynamic point of view as 3D islands are only possible at incomplete wetting, or, in other words, when the interfacial energy is greater than zero. ${ }^{8,41-44}$ As shown below, the adhesion of the atoms to the wetting layer is also distributed along the island in addition to the strain distribution and plays a more significant role than the latter. Due to the lattice misfit the atoms are displaced from their equilibrium positions in the bottoms of the potential troughs they should occupy at zero misfit. In this way the adhesion of the atoms to the substrate is stronger in the middle of the islands and weaker at the free edges. The average adhesion of an island of a finite size is thus weaker compared with that of an infinite monolayer. An interfacial boundary appears and the wetting of the island by the substrate (the wetting layer) is incomplete on the average. It is this incomplete wetting that drives the formation of dislocation-free 3D islands on the uniformly strained wetting layer.
In the present paper we make use of a more realistic interatomic potential which is characterized by its anharmonicity, in the sense that the repulsive branch is steeper than the attractive branch, and by its nonconvexity, which means that it possesses an inflection point beyond which its curvature becomes negative. Recently Tan and Lam have used a Mie potential to describe the mode of growth in a kinetic Monte Carlo procedure. ${ }^{45}$ However, these authors did not study the effect of misfit sign. Moreover, the distribution of the stress in the 3D islands has been studied again within the continuum elasticity theory. ${ }^{45} \mathrm{Yu}$ and Madhukar ${ }^{46}$ computed the energy and the distribution of strain in coherent Ge islands on $\mathrm{Si}(001)$ using a molecular dynamics coupled with the Stillinger-Weber potential ${ }^{47}$ but did not study the effect of anharmonicity in the general case.

The use of such a potential allows us to answer the question of why coherently strained 3D islands appear predominantly in compressed overlayers. Comparing the energies of mono- and multilayer islands allows us to make definite conclusions concerning the mechanism of formation and growth of the 3D islands, and the thermodynamic reason for the narrow size distribution. It turns out that there is a critical 2D island size above which monolayer islands become unstable against bilayer islands. Thus, as has been shown earlier by Stoyanov and Markov, ${ }^{48,6}$ Priester and Lannoo, ${ }^{28}$ and Chen and Washburn, ${ }^{31}$ the monolayer islands appear as necessary precursors for the formation of 3D islands. Beyond another critical size the bilayer islands become unstable against trilayer islands, etc. Thus the growth of 3D islands consists of consecutive transformations. As a result of each one of them the islands thicken by one monolayer. The critical size for the mono-bilayer transformation increases sharply with decrease of the lattice misfit, going asymptotically to infinity at some critical misfit. The monolayer islands are thus always stable against the multilayer islands below this critical misfit, which explains the necessity of large misfit in order to grow coherent 3D islands. The critical misfit in expanded overlayers is nearly twice as large in absolute value than that in compressed overlayers, which in turn explains why coherent 3D islanding is very rarely (if at all) observed in expanded overlayers.

The edge atoms are more weakly bound than the atoms in the middle of the islands. This is due to the weaker adhesion of the edge atoms to the wetting layer. Thus, the 2D-3D transformation takes place by transport of atoms from the edges of the monolayer islands, where they are weakly bound, on top of their surfaces to form islands on the upper layer where they are more strongly bound. ${ }^{6,48}$ This process is then repeated in the transformation of bilayer to trilayer islands, etc. The critical size for the 2D-3D transformation to occur is the thermodynamic reason for the narrow size distribution of the 3D islands.

In the case of expanded overlayers the atoms interact with each other through the weaker attractive branch of the potential and most of the atoms are not displaced from their equilibrium positions. The size effect is very weak, the average adhesion is sufficiently strong, and the critical sizes for 2D-3D transformation either do not exist or appear under extreme conditions of very large absolute value of the misfit. In any case MDs are introduced before the formation of bilayer islands. Either coherent monolayer islands are energeti- 


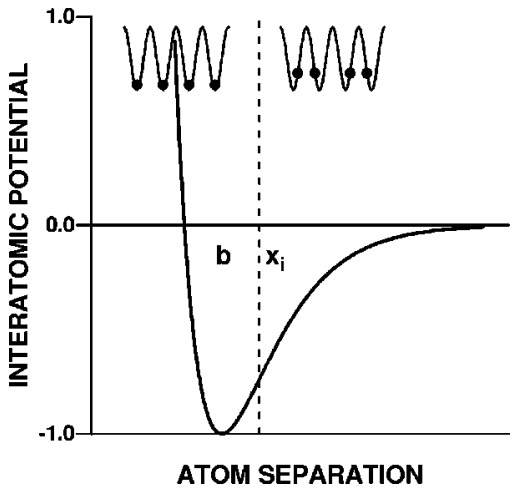

FIG. 2. The pairwise potential of Eq. (1) with $\mu=12, \nu=4$, and $V_{0}=1$. The dashed vertical line through the inflection point $x_{i}$ separates the regions of distortion $\left(x>x_{i}\right)$ and undistortion $\left(x<x_{i}\right)$ of the chemical bonds shown in the upper part of the figure.

cally stable against multilayer islands or MDs are introduced before the 2D-3D transformation. As a result the classical SK growth is expected in expanded overlayers.

\section{MODEL}

We consider a model in $1+1$ dimensions (substrate + height) which we treat as a cross section of the real $2+1$ case. An implicit assumption is that in the real $2+1$ case the monolayer islands have a compact rather than a fractal shape and the lattice misfit is one and the same in both orthogonal directions. Although the model is qualitative it gives correctly all the essential properties of the real $2+1$ system as shown by Snyman and van der Merwe. ${ }^{49-51}$ In this model the monolayer island is represented by a finite discrete FrenkelKontorova linear chain of atoms subject to an external periodic potential exerted by a rigid substrate (Fig. 4 below) ${ }^{52-54}$ We consider as a substrate a uniformly strained wetting layer of the same material consisting of an integer number of monolayers. In other words, we consider the SK growth in two separate stages. The first stage is a Frank-van der Merwe (layer-by-layer) growth during which the wetting layer is formed. The second stage is a Volmer-Weber growth of 3D islands on top of the wetting layer. In this paper we restrict ourselves to consideration of the second stage, assuming the wetting layer is already built up. The energetic influence of the initial substrate is already lost and the bonding between the atoms in the 3D islands is the same as that of the atoms of the first atomic plane of the 3D islands to the atoms belonging to the uppermost plane of the wetting layer.

The atoms of the chain are connected with bonds that obey the generalized Morse potential ${ }^{55-57}$

$$
V(x)=V_{0}\left[\frac{\nu}{\mu-\nu} e^{-\mu(x-b)}-\frac{\mu}{\mu-\nu} e^{-\nu(x-b)}\right]
$$

shown in Fig. 2 where $\mu$ and $\nu(\mu>\nu)$ are constants that govern the repulsive and the attractive branches, respectively, and $b$ is the equilibrium atom separation. For $\mu=2 \nu$ the potential (1) turns into the familiar Morse potential. In the case of homoepitaxy the bond strength $V_{0}$ is related to the energy barrier for desorption.

The potential (1) possesses an inflection point $x_{i n f}=b$ $+\ln (\mu / \nu) /(\mu-\nu)$ beyond which its curvature becomes nega- tive. This leads to a distortion of the interatomic bonds in the sense that long, weak and short, strong bonds alternate ${ }^{55-58}$ (see the upper right-hand corner of Fig. 2), and to the appearance of structures consisting of multiple MDs (multikink or kink-antikink-kink solutions). ${ }^{57}$ The latter represent two kinks (or solitons) connected by a strongly stretched out bond (the antikink).

The 3D islands can be represented by linear chains stacked one upon the other as in the model proposed by Stoop and van der Merwe ${ }^{59}$ and by Ratsch and Zangwill, ${ }^{34}$ each upper chain being shorter than the lower one. In principle, the Frenkel-Kontorova model is inadequate to describe a thickening overlayer because of two basic assumptions inherent in it. The first one is the rigidity of the substrate. Assuming that the substrate remains rigid upon formation of 3D islands on top of it rules out interaction between the islands through the elastic fields around them. It is believed that this assumption is valid for very thin deposits not exceeding one or two monolayers. The second one is connected with the relaxation effects. When a new monolayer island is formed on top of the previous one the latter should relax and the strains in the island will redistribute. One can expect that the formation, say, of a second monolayer will make the bonds between the first monolayer atoms effectively stiffer. As will be discussed below this will lead to weaker adherence of the atoms in the first monolayer to the wetting layer. MDs could also be introduced to relieve the strain. Nevertheless, the Frenkel-Kontorova model can provide excellent qualitative generalization in two dimensions both horizontally ${ }^{49-51}$ and vertically ${ }^{60}$ According to the authors of Ref. 60 an $n$-layer island can be mimicked by assuming that the force constant of the interatomic bonds is $n$ times greater than that of a monolayer island. Thus a bilayer island under compression could be simulated by doubling the value of the repulsive constant $\mu$. This approach obviously gives the upper bound of the effect of the next layers on the redistribution of the strain in the lower layers. An implicit shortcoming of this method is that it assumes the same number of bonds (and correspondingly atoms) in the upper chains and thus does not allow calculations of clusters with different slopes of the sidewalls.

Another approach to the problem has been proposed by Ratsch and Zangwill. ${ }^{34}$ They accepted that each layer (chain) presents a rigid sinusoidal potential to the chain of atoms on top of it. The atom, or, more precisely, the potential trough separation of the lower chain is taken as the average of all atom separations. As the strains of the bonds that are closer to the free ends are smaller, the average atom separation $b_{n}$ in the $n$th chain is closer to the unperturbed atom spacing $b$ and the lattice misfit $f_{n+1}=\left(b-b_{n}\right) / b_{n}$ for computing the energy of the $(n+1)$ st chain is smaller in absolute value than the misfit $f=(b-a) / a$, which is valid only for the base chain that is formed on the wetting layer, the latter having an atom separation $a$. In such a way the lattice misfit and in turn the bond strains gradually decrease with the island height. Every upper chain is taken to be shorter than the lower one by an arbitrary number of atoms and is centered on top of it as shown schematically in Fig. 3. Moreover, every uppermost chain is taken frozen (relaxation of the lower chain upon formation of the next one is ruled out) and serves as a template for the formation of the next one. Thus, the forma- 

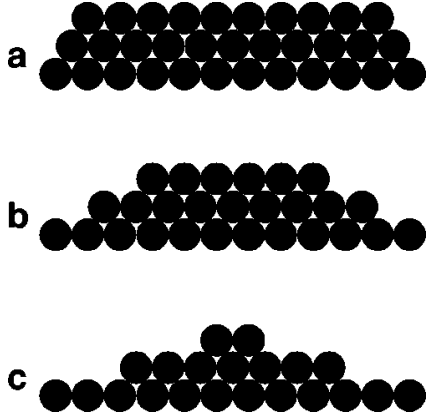

FIG. 3. Schematic view of multilayer islands with different slopes of the sidewalls: (a) $60^{\circ}$, (b) $30^{\circ}$, and (c) $19.1^{\circ}$.

tion of each next chain does not exert any influence on the distribution of strain in the previous chains, and this approach represents the lower bound of the effect of the next layer.

In the present paper we will use the approach of Ratsch and Zangwill. ${ }^{34}$ The main reason is that it allows a gradual attenuation of the strain with the island height, and also different angles of the sidewalls. We believe that although rather crude this approach gives correctly the essential physics with one exception. It does not account for the decrease of the average adhesion of the base chain to the wetting layer upon thickening of the islands. An approximate evaluation of the latter effect can be obtained by using the upper bound approach. It should be emphasized that both approaches show qualitatively identical results. We might expect that the results of more accurate calculations including the strain relaxation will not differ qualitatively by those presented below. Preliminary studies with an energy minimization program allowing strain relaxation always produced dislocated expanded and coherent compressed islands in agreement with the results shown below. Note that owing to the approximations of the model $(1+1$ dimensions and the lack of relaxation) the figures obtained as a result of the calculations, e.g., $3.25 \%$ for the critical misfit for 3D islanding, should not be taken as meaningful. Finally, we have to mention that the numerical solution of the system of governing equations (6) requires no more than a second on a $100 \mathrm{MHz}$ PC even when the number of equations (atoms in the chain) is about 100.

In discussing the stability of mono- and multilayer islands we follow the approach developed by Stoyanov and Markov. ${ }^{48}$ We start from the classical concept of the minimum of the surface energy at a fixed volume. Following Stranski, ${ }^{61}$ the surface energy $F(N)$ is defined as the difference between the potential energy of a cluster consisting of $N$ atoms and the potential energy of the same number of atoms in the bulk crystal,

$$
F(N)=N \phi_{k}-\sum_{i=1}^{N} \phi_{i},
$$

which is valid for clusters with arbitrary shape and size. Here $\phi_{k}$ is the work necessary to detach one atom from a kink position (or the energy of an atom in the bulk of the crystal), and the sum gives the work required to disintegrate the cluster into single atoms. Since the term $N \phi_{k}$ does not depend on the cluster shape the stability of mono- and multilayer islands is determined by the above sum. The maximum of the sum corresponds to a minimum of the surface (edge) energy of the cluster. Therefore, as a measure of stability, we adopt the potential energy per atom of the clusters, which is, in fact, equal to the above sum taken with a negative sign.

The potential energy of a chain of the $n$th layer consisting of $N_{n}$ atoms reads

$$
E_{n}=\sum_{i=1}^{N_{n}-1} V\left(X_{i+1}-X_{i}-b\right)+\sum_{i=1}^{N_{n}} \Phi_{i},
$$

where

$$
\Phi_{i}=\frac{W}{2}\left[1-\cos \left(2 \pi \frac{X_{i}}{b_{n-1}}\right)\right]
$$

accounts for the adhesion of the $i$ th atom. $X_{i}$ are the coordinates of the atoms taken from an arbitrary origin. The difference $\Delta X_{i}=X_{i+1}-X_{i}$ is in fact the distance between the ( $i$ $+1)$ st and $i$ th atoms. The first sum in Eq. (2) gives the energy of the bond strains. The second sum gives the energy of the atoms in the periodic potential field created by the lower chain, where $W$ is its amplitude and $b_{n-1}$ is the average potential trough separation of the underlying layer. In general, $W$ should be a function of the atom separation of the underlying layer and thus should depend on $n$, but for simplicity we neglect this dependence. As mentioned above $b_{n-1}=a$ holds only for the base chain. The amplitude $W$ can be considered in our model as the barrier for surface diffusion. On a nearest-neighbor bond hypothesis $W$ is related to the substrate-deposit bond strength by

$$
W=g V_{0},
$$

where $g<1$ is a constant of proportionality varying approximately from $1 / 30$ for long-range van der Waals forces to $1 / 3$ for short-range covalent bonds. ${ }^{62}$

The average of the second sum in Eq. (2) for the base chain divided by $V_{0}$,

$$
\Phi=\frac{1}{N_{1} V_{0}} \sum_{i=1}^{N_{1}} \Phi_{i},
$$

has the same physical meaning as the adhesion parameter

$$
\Phi=\frac{\sigma+\sigma_{i}-\sigma_{s}}{2 \sigma}=1-\frac{\beta}{2 \sigma}
$$

that accounts for the incomplete wetting of the 3D islands by the substrate in heteroepitaxy $\left(\sigma, \sigma_{i}\right.$, and $\sigma_{s}$ being the specific surface energies of the overlayer, the interface, and the substrate, respectively, and $\beta$ being the specific adhesion energy). ${ }^{8}$ In the case of classical SK growth the adhesion parameter is given by $\Phi=\epsilon_{d} / 2 \sigma$ where $\epsilon_{d}$ is the energy of a net of MDs. ${ }^{63}$ We have the case of complete wetting when $\Phi \leqslant 0$. The formation of $3 \mathrm{D}$ islands can obviously take place only when $0<\Phi<1$. $^{8}$

Minimization of $E_{n}$ with respect to $X_{i}$ results in a set of governing equations for the atom coordinates in the form

$$
e^{-\mu \epsilon_{i+1}}-e^{-\nu \epsilon_{i+1}}-e^{-\mu \epsilon_{i}}+e^{-\nu \epsilon_{i}}+A \sin \left(2 \pi \xi_{i}\right)=0,
$$

where $\epsilon_{i}=b_{n-1}\left(\xi_{i}-\xi_{i-1}-f_{n}\right)$ is the strain of the $i$ th bond, $\xi_{i}=X_{i} / b_{n-1}$ is the displacement of the $i$ th atom with respect 
(a)

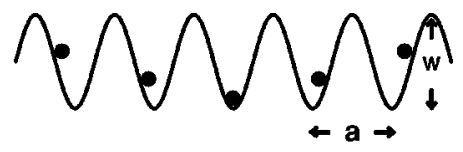

(b)

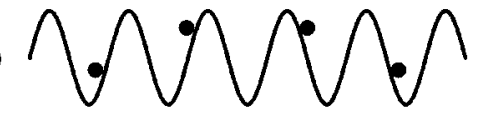

(c)

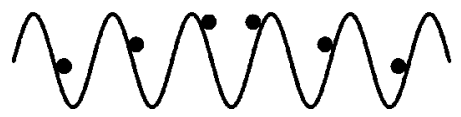

FIG. 4. Illustration of the solutions of the one-dimensional model of Frank and van der Merwe: (a) a chain without a misfit dislocation, (b) a misfit dislocation in a compressed chain, (c) a misfit dislocation in an expanded chain. With increasing chain length in (a) the end atoms are more displaced from the bottoms of the potential troughs and approach the crests between them.

to the bottom of the $i$ th potential trough, $f_{n}$ is the misfit between the $n$th chain and the substrate potential exerted by the $(n-1)$ st chain, and $A=\pi W(\mu-\nu) / \mu \nu b_{n-1} V_{0}$. The lattice misfit has its largest value $f=(b-a) / a$ only for the base chain in multilayer islands, and goes to zero with increasing island thickness. Expanding the exponentials in Taylor series for small strains gives the set of equations that govern the discrete harmonic model. ${ }^{53,54}$ Solving the system of equations (7) numerically gives the atom displacements $\xi_{i}$ and all the parameters characterizing the system can be easily computed.

The properties of the solutions of the system (7) are of crucial importance for understanding coherent SK growth. Two forces act on each atom: first is the force exerted by the neighboring atoms, and second is the force exerted by the substrate (the underlying chain or the wetting layer). The first force tends to preserve the natural spacing $b$ between the atoms, whereas the second force tends to place all the atoms at the bottoms of the corresponding potential troughs of the substrate separated at a distance $b_{n} \neq b$. As a result of the competition between the two forces the bond strains and the atom adhesion are distributed along the chain. The undislocated solution [Fig. 4(a)] clearly shows the decrease of the atom adhesion at the ends of the chain as the atoms are more and more displaced toward the chain ends. In the case of positive misfit the dislocation represents an empty potential trough, the bond in the core of the dislocation being strongly stretched out [Fig. 4(b)]. This picture is equivalent to a crystal plane in excess in the substrate. In the opposite case of negative misfit [Fig. 4(c)] the dislocation represents two atoms in one trough (a crystal plane in excess in the overlayer), the bond in the dislocation core being compressed. Both configurations are energetically equivalent in the harmonic approximation where the force between the atoms increases linearly with the atom separation. This is not, however, the case when an anharmonic potential is adopted. The latter displays a maximum force between the atoms at $x=x_{\text {inf }}$. This is the theoretical tensile stress of the material $\sigma_{\text {tens }}$ $=V_{0} \mu(\nu / \mu)^{\mu /(\mu-\nu)}$ and if the actual force exerted on the corresponding bond is greater than $\sigma_{\text {tens }}$ the bond will break..$^{55-57,64}$ Thus the interval of existence of dislocated solutions in compressed chains depends on the material param-
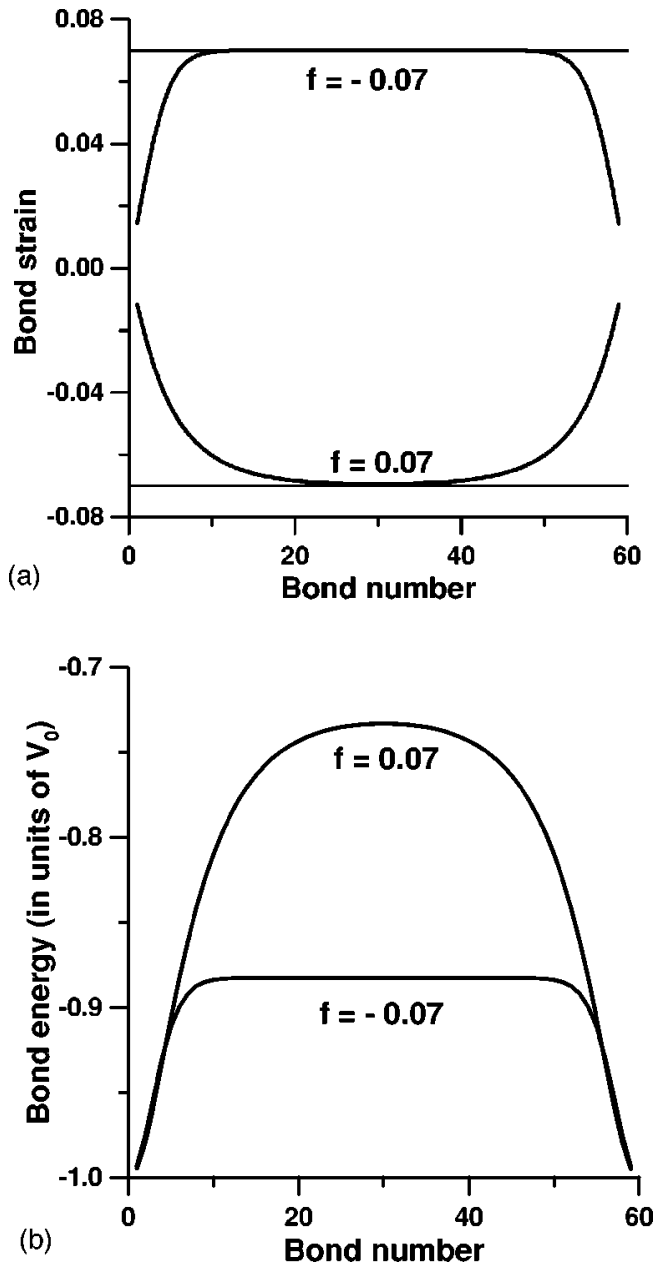

FIG. 5. Distribution (a) of the strain $\epsilon_{i}=\xi_{i+1}-\xi_{i}-f$ in monolayer height compressed $(f=0.07)$ and expanded $(f=-0.07)$ chains, and (b) of the corresponding bond energy in units of $V_{0}$. $W / V_{0}=1 / 3, \mu=12, \nu=6$.

eters $V_{0}, W, \mu, \nu, f$, and becomes very narrow. Dislocated solutions in compressed chains exist only in sufficiently long chains ${ }^{55-57}$ beyond some critical chain length. As will be shown below, this leads to coherent SK growth in compressed overlayers. On the contrary, the bonds in the cores of the MDs in expanded chains are compressed and cannot break. As a result MDs become energetically favored and can be introduced in very short chains. Thus, classical SK growth should be expected in expanded overlayers as dislocated islands with a monolayer height can become energetically favored long before coherently strained multilayer islands.

\section{RESULTS}

\section{A. Monolayer islands}

The distribution of the bond strains along the chains is shown in Fig. 5(a). As expected the bonds in the middle of the chains are strained to fit exactly the uniformly strained wetting layer. The strains at the chain ends tend to zero. In fact, the strains of the hypothetical zeroth and $N$ th bonds should be exactly equal to zero. ${ }^{53,54}$ The strains in the middle of the expanded chain compared with those of compressed ones are much closer to $-f$, owing to the weaker attraction 


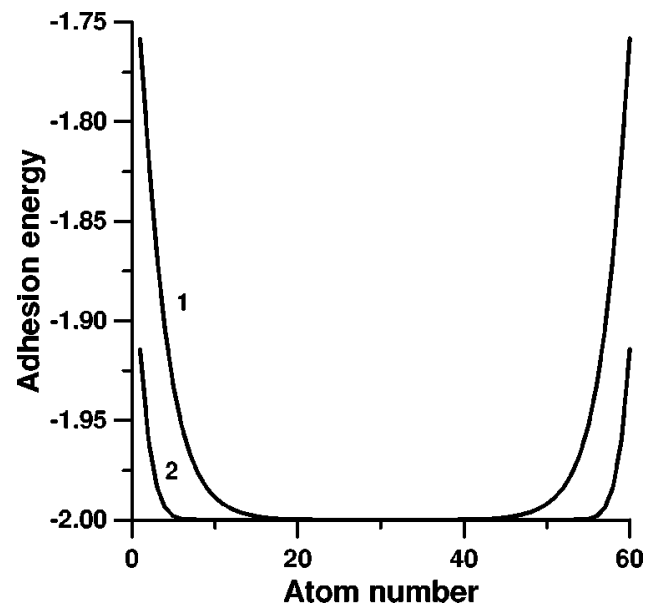

FIG. 6. Distribution of the adhesion energy $\Phi_{i} / V_{0}-1$ in monolayer height compressed (curve 1) and expanded (curve 2) chains. $W / V_{0}=1 / 3, \mu=12, \nu=6$.

between the atoms of the chain. Figure 5(b) shows the distribution of the bond energy. It is seen that in the case of compressed chains $(f>0)$ the bond energy in the middle of the chain is smaller than that in expanded chains owing to the stronger atom repulsion.

The distribution of the adhesion of the separate atoms $\Phi_{i}$ [Eq. (3)] [taken in terms of the bond energy $V_{0}$ as $\left(\Phi_{i}\right.$ $\left.\left.-V_{0}\right) / V_{0}\right]$ is demonstrated in Fig. 6. The weaker adhesion at the chain ends, which is often overlooked in theoretical models, is due to the displacement of the atoms from the bottoms of the potential troughs [see Fig. 4(a)]. What is more important is that the atoms in the expanded chains adhere much more strongly to the wetting layer compared with the atoms in the compressed chains.

Figure 7 shows the dependence of the mean adhesion parameter $\Phi[$ Eq. (5)] on the number of atoms. As can be expected the atom adhesion in expanded overlayers is stronger than that in compressed ones, owing to the weaker attraction between the atoms in the former. The forces exerted from the substrate are stronger than the forces between the chain atoms and the latter are situated more deeply in the potential troughs. The curves display maxima that are due to

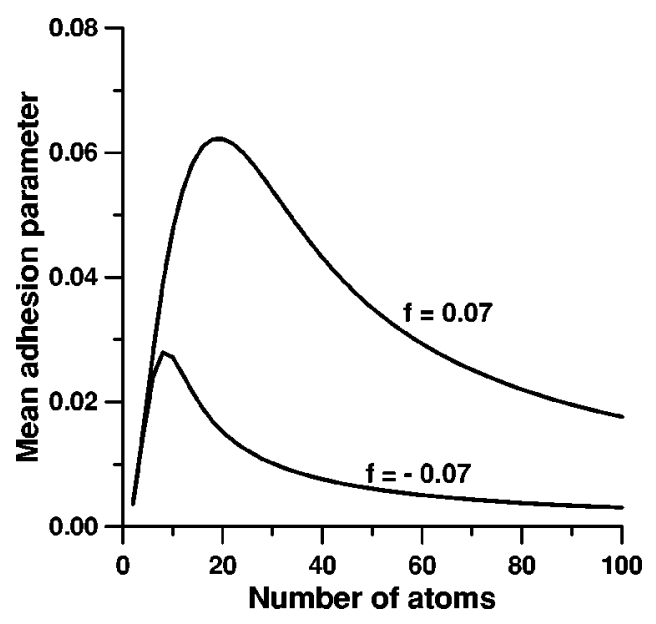

FIG. 7. The mean adhesion parameter $\Phi$ as a function of the number of atoms in the chains for positive $(f=0.07)$ and negative $(f=-0.07)$ values of the misfit. $W / V_{0}=1 / 3, \mu=12, \nu=6$.

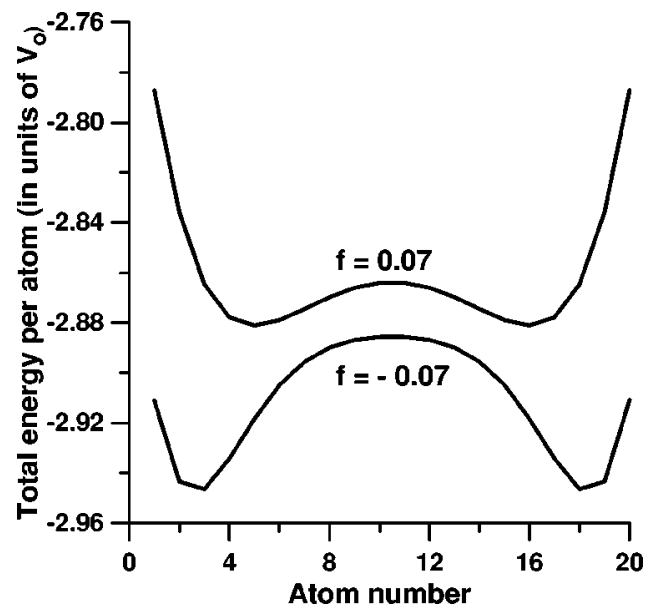

FIG. 8. Distribution of the total energy (strain plus adhesion) in units of $V_{0}$ in monolayer height compressed $(f=0.07)$ and expanded $(f=-0.07)$ chains. $W / V_{0}=1 / 3, \mu=12, \nu=6$.

the interplay between the fraction of the most strongly displaced end atoms and the values of the particular displacements. In short chains the atoms are weakly displaced from the bottoms of the potential troughs and the adhesion is stronger. With increasing chain length the displacements of the end atoms increase and beyond some length saturate and do not increase anymore. The fraction of weakly displaced middle atoms increases and a maximum is displayed. The value of the maximum (not shown) decreases sharply with decreasing misfit, going asymptotically to zero at zero misfit. This means that $\Phi>0$ at any value of nonzero misfit, which is the thermodynamic reason for $3 \mathrm{D}$ islanding.

Figure 8 shows the distribution of the total energy (strain plus adhesion) in chains with positive and negative misfit. The maxima in the middle are due to the strain contribution whereas the increase of the energy at the ends is due to the weaker adhesion. It is first seen that the atoms in the expanded chain are considerably more strongly bound to each other and to the substrate. The main difference between the two curves is that the atoms at the free ends in compressed chains are much more weakly bound than the end atoms in expanded chains. This result is of crucial importance for our understanding of the mechanism of transformation of monoto multilayer (3D) islands. We conclude from Fig. 8 that compressed islands display a greater tendency to transform into bilayer islands and further to form coherent 3D islands in comparison with expanded islands.

\section{B. Multilayer islands}

Multilayer (3D) islands can be full or frustums of pyramids and can have sidewalls with different slopes. The effect of the sidewall slope on the minimum energy shape is more or less clear. More unsaturated dangling bonds normal to the film plane appear on sidewalls with smaller slope and the corresponding surface energy is greater. Obviously, the surface energy of the steepest walls with a slope of $60^{\circ}$ is the lowest one. One might expect that the islands bounded with the steepest walls will be more stable than the flatter islands. The problem of whether the pyramids are full or frustums is more difficult to resolve. First, with increasing pyramid height the lattice misfit decreases and the mean strain van- 


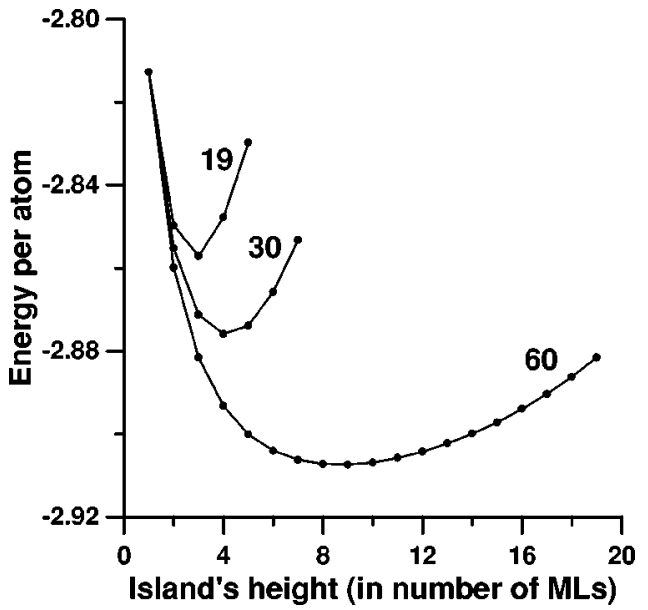

FIG. 9. Energy per atom of pyramidal 3D islands in units of $V_{0}$ with different slopes of the sidewalls denoted by the figures at each curve, as a function of their thickness in number of monolayers. The number of atoms $N_{1}=19$ in the base chain is one and the same for all curves. The frustum of a pyramid with a slope of $60^{\circ}$ of the sidewalls and height of 9 monolayers represents the equilibrium shape. $W / V_{0}=1 / 3, \mu=12, \nu=6$.

ishes. This in turn leads to increase of the adhesion of the separate atoms, and, as a whole, to an increase of the bond energy closer to the apex of the pyramids. On the other hand, the layers that are closer to the apex are smaller in size and the size effect increases. This leads to smaller work of evaporation per atom of the whole uppermost atomic plane. As has been known for a long time, the work required to disintegrate a whole atomic plane into single atoms (the mean separation work) taken with a negative sign is equal to its chemical potential at the absolute zero. ${ }^{4465}$ Hence, adding to the pyramid smaller and smaller upper base atomic planes leads to a decrease of the mean separation work of the upper base and in turn to higher chemical potential. As a result we might expect that frustums of pyramids with a slope of $60^{\circ}$ of the sidewalls will be energetically favored. This is clearly seen in Fig. 9, which demonstrates the energy per atom of pyramids with different sidewall slopes as a function of the height taken as the number of monolayers. The curves display minima at a certain height which clearly show that the frustums of pyramids are the lowest energy configurations. The energy of the full pyramids is much higher. The minimum of the $60^{\circ}$ sidewall slope is the lowest one, thus confirming the above consideration. The steepest sidewall slope of $60^{\circ}$ is a natural consequence of the model, which considers a face centered cubic rather than a diamond lattice. It is worth noting that Ratsch and Zangwill also report that the steepest sidewalls are energetically favored. ${ }^{34}$

The above result does not mean that in real experiments coherent 3D islands will grow as frustums of pyramids. The lowest minimum in Fig. 9 represents in fact the equilibrium shape of the islands. In reality, the crystallites grow with a shape that is determined by the rates of growth of the different walls and thus depends on the supersaturation. ${ }^{66}$ The growing crystal is bounded by the walls with the lowest growth rate at the given supersaturation. Mo et al. have established with the help of scanning tunneling microscopy (STM) that small coherently strained Ge islands ("hut" islands) grow on $\mathrm{Si}(001)$ as full pyramids bounded with (105) sidewalls, ${ }^{11}$ whereas Voigtländer and Zinner observed frustums of tetrahedral Ge pyramids on $\mathrm{Si}(111)$ with aspect (height-to-base) ratio showing a maximum of about 0.135 at a coverage of 4 monolayers (MLs). ${ }^{13}$ All the above is valid for sufficiently large crystals. We are interested here in the initial stages of growth of 3D islands, or, more precisely, in the transformation of monolayer into multilayer islands. As shown in the next sections, the formation and growth of 3D islands proceeds by consecutive transformations of monolayer islands into bilayer and then into multilayer islands, which is the lowest energy path of the 2D-3D transformation.

It should be stressed that the adhesion parameter $\Phi$ of a monolayer island should differ significantly from that of a multilayer island with the same base chain length. In our model they are equal. The reason is that the model does not allow the relaxation of the lower chains after formation of new ones on top of them. This is obviously incorrect as the formation of a second chain on top of the base one leads to effectively stronger lateral bonds in the bilayer islands. ${ }^{60} \mathrm{We}$ will try to evaluate this problem qualitatively and to discuss its consequences. As mentioned above the bilayer island could be treated as a first approximation as a monolayer island with a doubled force constant. ${ }^{60}$ As a result both the fraction of the strongly displaced end atoms and the corresponding displacements will be larger. Then the adhesion parameter of a bilayer island will be greater than that of a monolayer island with the same width. An evaluation of this effect can be made by using the approach of van der Merwe et al. mentioned above, ${ }^{60}$ by doubling of the constant $\mu$ in compressed chains. Thus for mono-, bi-, and trilayer islands with $\mu=12,24$, and 36 , one obtains $\Phi=0.024,0.066$, and 0.1 , respectively ( $\nu=6, f=0.05, N=21$ ). As seen the effect of the third layer is weaker than that of the second, which is easy to understand. The effect of formation of the next monolayers will have a smaller effect on the adhesion of the island and after some thickness the adhesion parameter will not change anymore. Thus the base layer atoms in a coherent multilayer island are more weakly bound to the wetting layer. What follows is that once formed the bilayer islands stabilize further growth of coherent 3D islands.

\section{Stability of mono- and multilayer islands}

We compare further the energies of mono- and multilayer islands with different thicknesses. The latter are bounded with $60^{\circ}$ sidewalls as they have the lowest minimum energy as shown above. Figure 10(a) shows the dependence of the energy of compressed monolayer and multilayer islands on the total number of atoms at a comparatively small lattice misfit of $3 \%$. As seen, the monolayer islands are always stable against bilayer and trilayer islands. A 2D-3D transformation is thus not expected and the film should continue to grow in a layer-by-layer mode coupled with the introduction of MDs at a later stage. The same dependence but at a larger misfit of 5\% is demonstrated in Fig. 10(b). The monolayer islands become unstable against the bilayer islands beyond a critical island size $N_{12}$, the bilayer islands in turn become unstable against the trilayer islands beyond a second critical number $N_{23}$, etc. The curve denoted by MD represents the energy of a monolayer chain containing one MD. The latter 

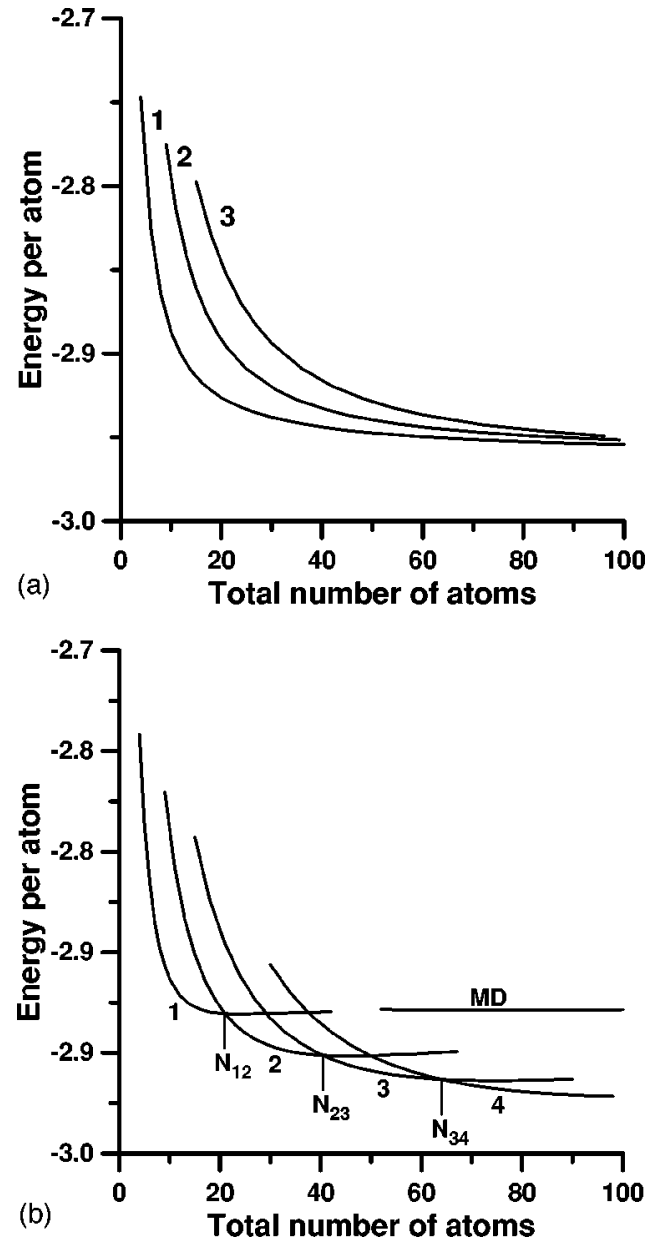

FIG. 10. The dependence of the energy per atom on the total number of atoms in compressed coherently strained islands with different thicknesses in monolayers denoted by the figures at each curve: (a) $f=0.03$, (b) $f=0.05$. The curve denoted by MD in (b) represents the energy of a monolayer chain containing one misfit dislocation. The numbers $N_{12}, N_{23}$, etc. give the limits of stability of monolayer, bilayer islands, respectively. $W / V_{0}=1 / 3, \mu=12$, $\nu=6$.

begins at a large number of atoms $(N=52)$ because the bonds in the cores of the MDs break up for shorter chains. This is due to the fact that the force exerted on these bonds from the neighboring atoms is greater than the theoretical tensile stress of the film material $\sigma_{\text {tens }}$, as mentioned above. Curve 1, which represents the energy of the undislocated monolayer chain, is computed for clarity up to a number of atoms smaller than the number (52) at which the solutions of the dislocated chain appear. The reason is that the values of the energy are very close and the curves are undistinguishable to the eye. The energies of monolayer chains with and without MDs cross each other at about $N=300$ (not shown) which means that coherent $3 \mathrm{D}$ islands are formed long before the introduction of MDs. Moreover, the dislocated chain with a monolayer height has an energy much higher than the energies of the undislocated multilayer islands. This clearly shows that the film "prefers" to grow as coherent 3D islands in which the gradual decrease of the strain energy overcompensates the surface energy, rather than to introduce MDs in the first monolayer.

Figure 11 demonstrates the same dependence as in Fig. 10

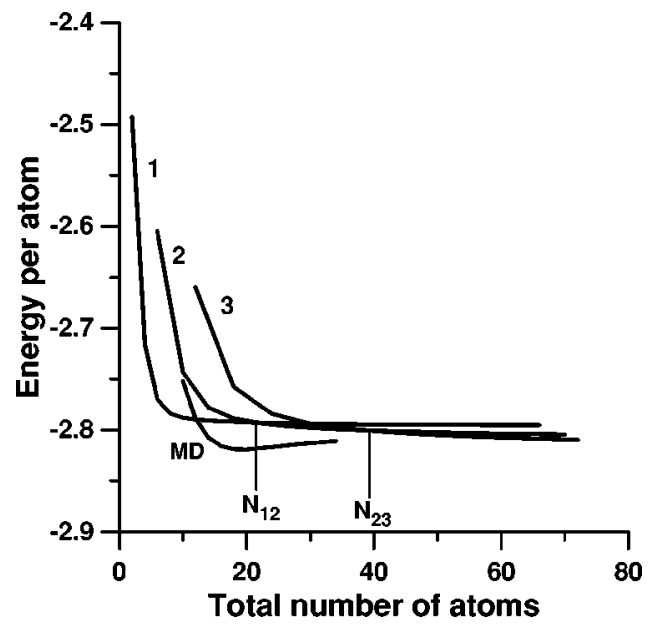

FIG. 11. The dependence of the energy per atom on the total number of atoms in units of $V_{0}$ in expanded coherently strained islands with different thickness in monolayers denoted by the figures at each curve, and at large negative value of the misfit $f$ $=-0.1$. The curve denoted by MD represents the energy of a monolayer chain containing one misfit dislocation. $W / V_{0}=1 / 3, \mu$ $=12, \nu=6$.

but in expanded chains. The absolute value of the negative misfit is very large $(-10 \%)$. At absolute values of the misfit smaller than $5.5 \%$ (not shown) the behavior of the energies is the same as in Fig. 10(a). The energies of the coherent monoand multilayer chains cross each other again at some critical number of atoms but the dislocated monolayer chain (denoted by MD) becomes energetically favored noticeably before the coherent bilayer chain becomes stable. Classical SK growth should take place in expanded overlayers.

Figure 12 shows the misfit dependence of the first critical size $N_{12}$ for both positive and negative misfits. As seen, it increases sharply with decreasing misfit, going asymptotically to infinity at some critical misfits denoted by the vertical dashed lines. The existence of a critical positive misfit for coherent SK growth to occur explains why high mismatch epitaxy is required in order to grow coherent 3D islands. The critical misfit below which the expanded monolayer islands

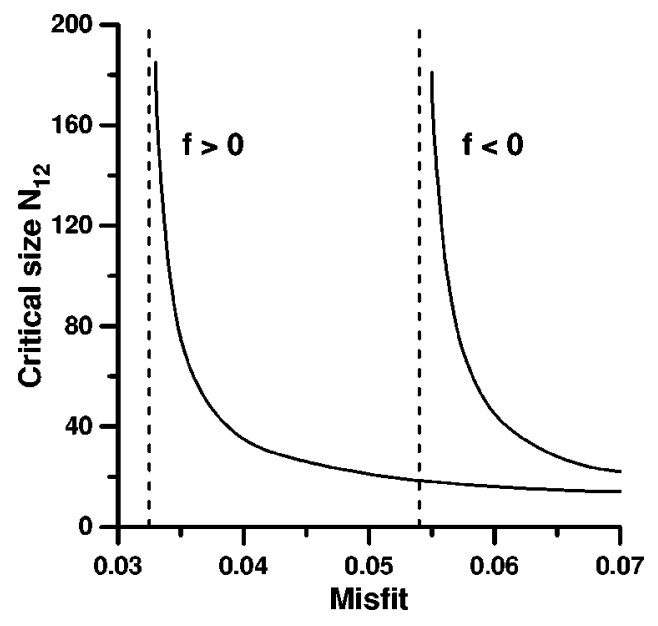

FIG. 12. Misfit dependence of the critical size $N_{12}$. The vertical dashed lines denote the critical misfits below which $N_{12}$ is infinite. The curves are shown in one quadrant for easier comparison. 


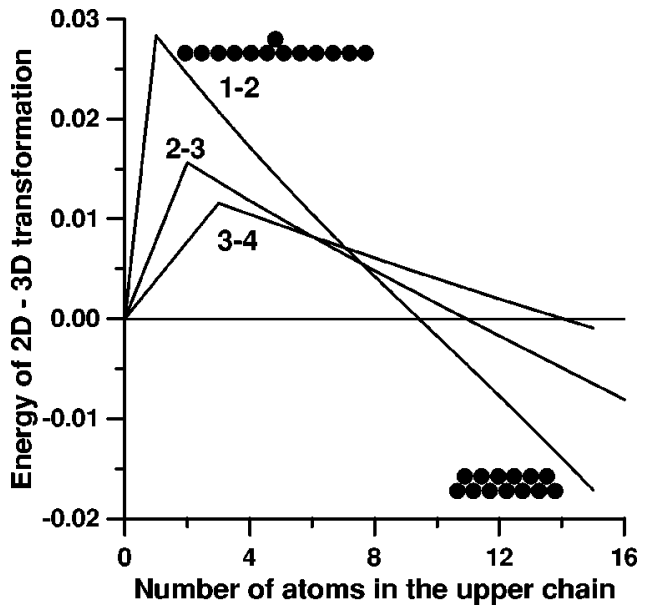

FIG. 13. The energy change $\Delta E_{n}$ in units of $V_{0}$ connected with the transformation of mono- to bilayer islands (curve 1-2), bi- to trilayer islands (curve 2-3), and tri- to four-layer islands (curve 3-4), as a function of the number of atoms $n$ in the uppermost chain. $f$ $=0.05, W / V_{0}=1 / 3, \mu=12, \nu=6$.

are always stable against multilayer islands is nearly twice as large in absolute value compared with the same quantity in compressed overlayers. Thus coherent SK growth in expanded overlayers could be observed at unrealistically large absolute values of the negative misfit.

We conclude that classical SK growth or 2D growth will be observed in the thermodynamic limit at small positive misfits and coherent SK growth at misfits greater than a critical misfit. This result clearly explains why large positive misfit is required for coherent SK growth to occur. The large positive misfit leads to large atom displacements and in turn to weaker adhesion. The physics is essentially the same as in the case of heteroepitaxial growth of 3D islands directly on top of the surface of the foreign substrate (Volmer-Weber growth). ${ }^{48}$

\section{Mechanism of 2D-3D transformation}

It is natural to assume that once the monolayer islands become unstable against the bilayer islands $\left(N>N_{12}\right)$, the former should rearrange themselves into bilayer islands. As shown below the mono-bilayer transformation can be considered as the first step for building sufficiently high 3D crystallites. The mechanism of the mono-bilayer transformation is easy to predict, having in mind that the edge atoms are more weakly bound than the atoms in the middle. The edge atoms can detach and diffuse on top of the monolayer islands, giving rise to clusters in the second layer. We consider first in more detail the transformation of a monolayer island (chain) with a length $N_{0}>N_{12}$ into a bilayer island. For this aim we plot the energy $E(n)$ of an incomplete bilayer island which consists of $N_{0}-n$ atoms in the lower layer and $n$ atoms in the upper layer referred to the energy $E_{0}$ of the initial chain consisting of $N_{0}$ atoms, as a function of the number of atoms $n$ in the upper layer. This is the curve denoted by 1-2 in Fig. 13. As seen, it displays a maximum at $n=1$ after which $\Delta E_{n}=E(n)-E_{0}$ decreases up to the complete mono-bilayer transformation, at which $n=\left(N_{0}-1\right) / 2$.

Curve 1-2 in Fig. 13 has the characteristic behavior of a nucleation process. The cluster at which the maximum of $\Delta E$ is observed can be considered as the critical nucleus of the second layer. As shown in Ref. 48, the mono-bilayer transformation is a real nucleation process when the $2+1$ heteroepitaxial Volmer-Weber model is considered, in other words, when the 3D islands are formed directly on top of the foreign substrate without the formation of an intermediate wetting layer. The chemical potential of the upper island at the maximum is exactly equal to that of the initial monolayer island, and the supersaturation with which the nucleus of the second layer is in equilibrium is equal to the difference of the energies of desorption of the atoms from the same and the foreign substrate. This is, namely, the driving force for the 2D-3D transformation to occur. The $1+1$ model is in fact one dimensional and the nuclei do not exist in the thermodynamic sense because the length of a row of atoms does not depend on the supersaturation. ${ }^{44,67}$ However, considering our $1+1$ model as a cross section of the real $2+1$ case, we can treat the curve 1-2 in Fig. 13 as the size dependence of the free energy for nucleus formation and growth. We would like to emphasize that in the $2+1$ case the nucleus does not necessarily consist of one atom. Its size should depend on the lattice misfit, and in a real situation on the temperature. The curves denoted by 2-3 and 3-4 in Fig. 13 represent the energy changes of bilayer to trilayer islands, and of trilayer to four-layer islands, respectively. As seen, they behave in the same way and the work for nucleus formation (the respective maxima) decreases with thickening of the islands. This means that the mono-bilayer transformation is the rate determining process for the total mono-multilayer (2D-3D) transformation.

\section{DISCUSSION}

The Stranski-Krastanov growth mode appears as a result of the interplay of the film-substrate bonding, strain, and surface energies. A wetting layer is first formed on top of which 3D islands nucleate and grow. The 3D islands and the wetting layer represent necessarily different phases. If this was not the case the growth would continue by 2D layers. Thus we can consider as a useful approximation the 3D islanding on top of a uniformly strained wetting layer to be Volmer-Weber growth. That requires the adhesion of the atoms to the substrate to be smaller than the cohesion between the overlayer atoms. In other words, the wetting of the substrate by the overlayer should be incomplete. In classical SK growth this condition is fulfilled because of the formation of an array of misfit dislocations at the boundary between the islands and the wetting layer. The atoms are displaced from the bottoms of the potential troughs [mostly in the cores of the MDs, see Figs. 4(b) and 4(c)] and thus are more weakly bound on average to the underlying wetting layer, irrespective of the fact that the chemical bonding is one and the same. As a result the lattice misfit gives rise to an effective adhesion that is weaker than the cohesion of the overlayer atoms. In contrast to the wetting layer, the 3D islands are elastically relaxed and their atom density differs from that of the former. Thus, the wetting layer and the 3D islands really represent different phases separated by a clear interfacial boundary, whose energy is in fact the energy of the array of MDs. The physical reason for 3D islanding in coherent SK growth is essentially the same. In this case the atoms near the 
island edges are displaced from the bottoms of the corresponding potential troughs [see Fig. 4(a)] and they adhere more weakly to the wetting layer compared with the atoms in the middle. The thicker the islands the stronger is this tendency. Thus, the average adhesion of the 3D islands to the wetting layer is again weaker than the cohesion in the islands themselves. Thus we can treat the coherent SK growth as Volmer-Weber growth on top of the wetting layer. The main difference is that in Volmer-Weber growth the adhesion parameter $\Phi$ is constant whereas in the coherent SK mode it depends on the island thickness.

The weaker adhesion means in fact an incomplete wetting, which appears as the thermodynamic driving force for 3D islanding. The smaller the misfit the smaller are the displacements of the edge atoms and in turn the stronger is the average wetting. The latter leads to the appearance of a critical misfit below which the edge effects do not play a significant role. The average wetting is very strong and the formation of coherent 3D islands becomes thermodynamically unfavored. The film will continue to grow in a $2 \mathrm{D}$ mode until the strain is relaxed by introduction of MDs or dislocated 3D islands at a later stage. The existence of a critical misfit for the 2D-3D transformation to occur both in compressed and expanded overlayers has been noticed in several studies. Pinczolits et al. ${ }^{27}$ have found that deposition of $\mathrm{PbSe}_{1-x} \mathrm{Te}_{x}$ on $\mathrm{PbTe}(111)$ remains purely two dimensional when the misfit is less than $1.6 \%$ in absolute value (Se content $<30 \%$ ). Leonard et $a .^{3}$ have successfully grown quantum dots of $\mathrm{In}_{x} \mathrm{Ga}_{1-x} \mathrm{As}$ on $\operatorname{GaAs}(001)$ with $x=0.5(f \approx 3.6 \%)$ but $60 \AA$ thick 2D quantum wells at $x=0.17(f \approx 1.2 \%)$. A critical misfit of $1.4 \%$ has been found by Xie et al. upon deposition of $\mathrm{Si}_{0.5} \mathrm{Ge}_{0.5}$ films on relaxed buffer layers of $\mathrm{Si}_{x} \mathrm{Ge}_{1-x}$ with varying composition. $^{68}$

The average adhesion (the wetting) depends strongly on the anharmonicity of the interatomic forces. Expanded islands adhere more strongly to the wetting layer and the critical misfit beyond which coherent $3 \mathrm{D}$ islanding is possible is much greater in absolute value compared with that in compressed overlayers. As a result coherent SK growth in expanded films could be expected at very (unrealistically) large absolute values of the negative misfit. The latter, however, depends on the material parameters (degree of anharmonicity, strength of the chemical bonds, etc.) of the particular system and cannot be completely ruled out. Xie et al. ${ }^{68}$ studied the deposition of $\mathrm{Si}_{0.5} \mathrm{Ge}_{0.5}$ films in the whole range of $2 \%$ tensile misfit to $2 \%$ compressive misfit on relaxed buffer layers of $\mathrm{Si}_{x} \mathrm{Ge}_{1-x}$ starting from $x=0$ (pure $\mathrm{Ge}$ ) to $x=1$ (pure $\mathrm{Si}$ ), and found that 3D islands are formed only under compressive misfit larger than $1.4 \%$. Films under tensile strain were thus stable against 3D islanding, in excellent agreement with the predictions of our model.

The weaker average adhesion in compressed overlayers leads to another effect at misfits greater than the critical one. At some critical number of atoms $N_{12}$ the monolayer islands become unstable against bilayer islands. The latter become in turn unstable against trilayer islands beyond another critical number $N_{23}$, and so on. As a result, the complete 2D-3D transformation should take place during growth by consecutive transformations of mono- to bilayer, bi- to trilayer islands, etc. Owing to the stronger interatomic repulsive forces the edge atoms in the compressed monolayer islands adhere more weakly to the wetting layer compared with the edge atoms in expanded islands. This results in an easier transformation of mono- to bilayer islands, which is the first step to the complete 2D-3D transformation. The latter includes also kinetics in the sense that the edge atoms have to detach and form the upper layers. However, it is not the strain at the edges (which is nearly zero) that is responsible for the easier detachment of the edge atoms as suggested by Kandel and Kaxiras $^{69}$ but the weaker adhesion. The 2D-3D transformation is hindered in expanded islands as the edge atoms adhere more strongly to the wetting layer. On the other hand, the existence of such critical sizes, which determine the intervals of stability of islands with different thicknesses, could be considered as the thermodynamic reason for the narrow size distribution of 3D islands which is observed in experiments. This does not mean that this is the only reason. Elastic interactions between islands and growth kinetics can have greater effects than thermodynamics. The 2D-3D transformation takes place by consecutive nucleation events, each one needing to overcome a lower energetic barrier than the one before. Thus, the mono-bilayer transformation appears as the rate determining process.

Let us consider all the above from another point of view. The results displayed in Fig. 10(b) show that the equilibrium shape aspect ratio increases gradually with the island volume. The consecutive stability of islands with increasing thickness reflects the simple fact that the increase of the pyramid height is discrete (layer after layer) whereas the base chain length remains nearly constant. The stronger the adhesion or the smaller the misfit the wider will be the intervals of stability of islands with a fixed height, and vice versa. The formation of every new crystal plane on the upper crystal face requires the appearance of a 2D nucleus. As the growing surface is usually very small, the formation of one nucleus is sufficient for the growth of a new crystal plane. Thus we could expect a mononucleus layer-by-layer growth of the pyramids. ${ }^{4,66}$ This has been independently established by using a kinetic Monte Carlo method by Khor and Das Sarma. ${ }^{70}$ It should be noted that Duport, Priester, and Villain established that the monolayer islands are thermodynamically favored up to a critical size, beyond which the equilibrium shape becomes nearly a full pyramid. ${ }^{37}$ The transition from a monolayer island to a pyramid is of first order and requires the overcoming of an activation barrier which is proportional to $f^{-4}$.

It should be stressed that our definition of the critical 2D island size $N_{12}$ for 2D-3D transformation to begin differs from that in the papers of Priester and Lannoo ${ }^{28}$ and Chen and Washburn. ${ }^{31}$ The former authors define the critical size by comparing the energy per atom of monolayer islands with that of fully built 3D pyramids. Chen and Washburn have accepted as critical the size at which the energy of the monolayer islands displays a minimum. ${ }^{31}$ They found also that the critical size $N_{c}$ determined by the minimum of the energy increases very steeply with decreasing misfit $\left(N_{c} \propto f^{-6}\right)$. Although our definition of $N_{c}$ is different we also observe a very steep misfit dependence (see Fig. 12).

A rearrangement of monolayer height (2D) islands into multilayer (3D) islands has been reported by Moison et al. ${ }^{19}$ who established that InAs 3D islands begin to form on GaAs at a coverage of about $1.75 \mathrm{ML}$ but then the coverage sud- 
denly decreases to $1.2 \mathrm{ML}$. This decrease of the coverage in the second monolayer could be interpreted as a rearrangement of an amount of nearly half a monolayer into 3D islands. The same phenomenon has been noticed by Shklyaev, Shibata, and Ichikawa in the case of $\mathrm{Ge} / \mathrm{Si}(111){ }^{71}$ Voigtländer and Zinner noted that $\mathrm{Ge} 3 \mathrm{D}$ islands in $\mathrm{Ge} / \mathrm{Si}(111)$ epitaxy have been observed at the same locations where 2D islands locally exceeded the critical wetting layer thickness of two bilayers. ${ }^{13}$

Contrary to the linear theory of elasticity, the anharmonicity and nonconvexity of real interatomic potentials lead to different intervals of existence of misfit dislocations in compressed and expanded overlayers. The nonconvexity of the interatomic potential gives rise to the possibility of breaking the expanded bonds in the cores of the MDs in compressed overlayers when the force exerted on them is greater than the theoretical tensile strength of the material. As a result, MDs in compressed overlayers appear in sufficiently large islands and small coherent 3D islands can appear before that. On the contrary, this restriction does not exist in expanded overlayers where the bonds in the cores of the MDs are compressed. The introduction of MDs can thus become energetically favored in short chains (small islands) before the formation of coherent 3D islands, and classical SK growth should be observed in most cases.

It should be noted that the results presented above depend on the approximations of the model, particularly when the energy of the multilayer islands is computed. Allowing a strain relaxation of lower layers when new layers are formed on top of them could lead to earlier introduction of MDs but also to weaker adhesion of the 3D islands to the wetting layer. Thus, applying a more refined approach, which accounts for the strain relaxation in the islands, as well as in the wetting layer, will allow us to study the transition from the coherent to the classical (dislocated) Stranski-Krastanov growth mode.

In summary, accounting for the anharmonicity and the nonconvexity of real interatomic potentials in a model in $1+$ 1 dimensions, we have shown that coherent 3D islands can be formed on the wetting layer in the SK mode predominantly in compressed overlayers at sufficiently large values of the misfit. Coherent 3D islanding in expanded overlayers could be expected as an exception rather than as a rule. Monolayer height islands with a critical size appear as necessary precursors of the 3D islands. This explains the narrow size distribution of the 3D islands from the thermodynamic point of view.

\section{ACKNOWLEDGMENTS}

One of the authors (E.K.) is financially supported partly by the Spanish DGES Contract No. PB97-0076 and partly by Contract No. F608 of the Bulgarian National Fund for Scientific Research. I.M. gratefully acknowledges fruitful discussions with R. Kaischew. The authors greatly benefited from the remarks and criticism of Jacques Villain.
*Permanent address: G. Nadjakov Institute of Solid State Physics, Bulgarian Academy of Sciences, 1784 Sofia, Bulgaria.

'Author to whom correspondence should be addressed. FAX: $\quad(+359) \quad 2 \quad 971$ 2688. Electronic address: imarkov@ipchp.ipc.bas.bg

${ }^{1}$ For a recent review, see W. Seifert, N. Carlsson, M. Miller, M.-E. Pistol, L. Samuelson, and L. R. Wallenberg, Prog. Cryst. Growth Charact. Mater. 33, 423 (1996).

${ }^{2}$ D. J. Eaglesham and M. Cerullo, Phys. Rev. Lett. 64, 1943 (1990).

${ }^{3}$ D. Leonard, M. Krishnamurthy, C. M. Reaves, S. P. Denbaars, and P. M. Petroff, Appl. Phys. Lett. 63, 3203 (1993).

${ }^{4}$ Vinh Le Thanh, P. Boucaud, D. Débarre, Y. Zheng, D. Bouchier, and J.-M. Lourtioz, Phys. Rev. B 58, 13115 (1998).

${ }^{5}$ R. Kern, G. LeLay, and J. J. Metois, in Current Topics in Material Science, edited by E. Kaldis (North-Holland, Amsterdam 1979), Vol. 3, p. 128.

${ }^{6}$ I. Markov and S. Stoyanov, Contemp. Phys. 28, 267 (1987).

${ }^{7}$ J. W. Gibbs, The Collected Works of J. Willard Gibbs (Longmans, Green \& Co., New York, 1928).

${ }^{8}$ E. Bauer, Z. Kristallogr. 110, 372 (1958).

${ }^{9}$ P. Ashu and C. C. Matthai, Appl. Surf. Sci. 48/49, 39 (1991).

${ }^{10}$ B. G. Orr, D. Kessler, C. W. Snyder, and L. Sander, Europhys. Lett. 19, 33 (1992).

${ }^{11}$ Y.-W. Mo, D. E. Savage, B. S. Swartzentruber, and M. Lagally, Phys. Rev. Lett. 65, 1020 (1990).

${ }^{12}$ C. E. Aumann, Y.-W. Mo, and M. G. Lagally, Appl. Phys. Lett. 59, 1061 (1991).

${ }^{13}$ B. Voigtländer and A. Zinner, Appl. Phys. Lett. 63, 3055 (1993).

${ }^{14}$ F. K. LeGoues, M. C. Reuter, J. Tersoff, M. Hammar, and R. M. Tromp, Phys. Rev. Lett. 73, 300 (1994).
${ }^{15}$ F. M. Ross, J. Tersoff, and R. M. Tromp, Phys. Rev. Lett. 80, 984 (1998).

${ }^{16}$ M. Kästner and B. Voigtländer, Phys. Rev. Lett. 82, 2745 (1999).

${ }^{17}$ R. Heitz, T. R. Ramachandran, A. Kalburge, Q. Xie, I. Mukhametzhanov, P. Chen, and A. Madhukar, Phys. Rev. Lett. 78, 4071 (1997).

${ }^{18}$ N. P. Kobayashi, T. R. Ramachandran, P. Chen, and A. Madhukar, Appl. Phys. Lett. 68, 3299 (1996).

${ }^{19}$ J. M. Moison, F. Houzay, F. Barthe, L. Leprince, E. André, and O. Vatel, Appl. Phys. Lett. 64, 196 (1994).

${ }^{20}$ H. Yamaguchi, J. G. Belk, X. M. Zhang, J. L. Sudijono, M. R. Fahy, T. S. Jones, D. W. Pashley, and B. A. Joyce, Phys. Rev. B 55, 1337 (1997).

${ }^{21}$ B. A. Joyce, J. L. Sudijono, J. G. Belk, H. Yamaguchi, X. M. Zhang, H. T. Dobbs, A. Zangwill, D. D. Vvedensky, and T. S. Jones, Jpn. J. Appl. Phys., Part 1 36, 4111 (1997).

${ }^{22}$ A. Polimeni, A. Patane, M. Capizzi, F. Martelli, L. Nasi, and G. Salviati, Phys. Rev. B 53, R4213 (1996).

${ }^{23}$ S. Guha, A. Madhukar, and K. C. Rajkumar, Appl. Phys. Lett. 57, 2110 (1990).

${ }^{24}$ C. W. Sneider, B. G. Orr, D. Kessler, and L. M. Sander, Phys. Rev. Lett. 66, 3032 (1991).

${ }^{25}$ J. Temmyo, R. Nötzel, and T. Tamamura, Appl. Surf. Sci. 121/ 122, 63 (1997).

${ }^{26}$ N. Carlsson, W. Seifert, A. Peterson, P. Castrillo, M. E. Pistol, and L. Samuelson, Appl. Phys. Lett. 65, 3093 (1994).

${ }^{27}$ M. Pinczolits, G. Springholz, and G. Bauer, Appl. Phys. Lett. 73, 250 (1998)

${ }^{28}$ C. Priester and M. Lannoo, Phys. Rev. Lett. 75, 93 (1995).

${ }^{29}$ H. M. Koduvely and A. Zangwill, Phys. Rev. B 60, R2204 (1999). 
${ }^{30}$ B. J. Spencer, P. W. Voorhees, and S. H. Davis, Phys. Rev. Lett. 67, 3696 (1991).

${ }^{31}$ Y. Chen and J. Washburn, Phys. Rev. Lett. 77, 4046 (1996).

${ }^{32}$ J. Tersoff and R. M. Tromp, Phys. Rev. Lett. 70, 2782 (1993).

${ }^{33}$ J. Tersoff and F. K. LeGoues, Phys. Rev. Lett. 72, 3570 (1994).

${ }^{34}$ C. Ratsch and A. Zangwill, Surf. Sci. 293, 123 (1993).

${ }^{35}$ V. A. Shchukin, N. N. Ledentsov, P. S. Kop'ev, and D. Bimberg, Phys. Rev. Lett. 75, 2968 (1995).

${ }^{36}$ I. Daruka and A.-L. Barabási, Phys. Rev. Lett. 79, 3708 (1997).

${ }^{37}$ C. Duport, C. Priester, and J. Villain, in Morphological Organization in Epitaxial Growth and Removal, Vol. 14 of Directions in Condensed Matter Physics, edited by Z. Zhang and M. Lagally (World Scientific, Singapore, 1998).

${ }^{38}$ B. J. Spencer and J. Tersoff, Phys. Rev. Lett. 79, 4858 (1997).

${ }^{39}$ B. J. Spencer, Phys. Rev. B 59, 2011 (1999).

${ }^{40}$ L. G. Wang, P. Kratzer, M. Scheffler, and N. Moll, Phys. Rev. Lett. 82, 4042 (1999).

${ }^{41}$ I. N. Stranski, Z. Phys. Chem. Abt. A 142, 453 (1929).

${ }^{42}$ I. N. Stranski and K. Kuleliev, Z. Phys. Chem. Abt. A 142, 467 (1929).

${ }^{43}$ R. Kaischew, Fortschr. Mineral. 38, 7 (1960).

${ }^{44}$ For a review, see I. Markov, Crystal Growth for Beginners, Fundamentals of Nucleation, Crystal Growth and Epitaxy (World Scientific, Singapore, 1995).

${ }^{45}$ Sovirith Tan and Pui-Man Lam, Phys. Rev. B 60, 8314 (1999).

${ }^{46}$ W. Yu and A. Madhukar, Phys. Rev. Lett. 79, 905 (1997); 79, 4939 (1997).

${ }^{47}$ F. H. Stillinger and T. A. Weber, Phys. Rev. B 31, 5262 (1985).

${ }^{48}$ S. Stoyanov and I. Markov, Surf. Sci. 116, 313 (1982).

${ }^{49}$ J. A. Snyman and J. H. van der Merwe, Surf. Sci. 42, 190 (1974).

${ }^{50}$ J. A. Snyman and J. H. van der Merwe, Surf. Sci. 45, 619 (1974).

${ }^{51}$ J. A. Snyman and H. C. Snyman, Surf. Sci. 105, 357 (1981).
${ }^{52}$ Ya. I. Frenkel and T. Kontorova, J. Phys. Acad. Sci. USSR 1, 137 (1939).

${ }^{53}$ F. C. Frank and J. H. van der Merwe, Proc. R. Soc. London, Ser. A 198, 205 (1949).

${ }^{54}$ F. C. Frank and J. H. van der Merwe, Proc. R. Soc. London, Ser. A 198, 216 (1949).

${ }^{55}$ I. Markov and A. Trayanov, J. Phys. C 21, 2475 (1988).

${ }^{56}$ I. Markov and A. Trayanov, J. Phys.: Condens. Matter 2, 6965 (1990).

${ }^{57}$ I. Markov, Phys. Rev. B 48, 14016 (1993).

${ }^{58}$ C. Haas, Solid State Commun. 26, 709 (1978)

${ }^{59}$ L. C. A. Stoop and J. H. van der Merwe, Thin Solid Films 17, 291 (1973).

${ }^{60}$ J. H. van der Merwe, J. Woltersdorf, and W. A. Jesser, Mater. Sci. Eng. 81, 1 (1986).

${ }^{61}$ I. N. Stranski, Ann. Univ. Sofia (Livre 2, Chimie) 30, 367 (1936/ 1937).

${ }^{62}$ J. H. van der Merwe, Treatise on Materials Science and Technology (Academic Press, New York, 1973), Vol. 2, p. 1.

${ }^{63}$ I. Markov, Mater. Chem. Phys. 9, 93 (1983).

${ }^{64}$ I. Markov and A. Trayanov, J. Cryst. Growth 141, 239 (1994).

${ }^{65}$ I. N. Stranski and R. Kaischew, Phys. Z. 11, 393 (1935).

${ }^{66}$ A. A. Chernov, Modern Crystallography III (Springer, Berlin, 1984).

${ }^{67}$ I. N. Stranski, Z. Phys. Chem. 36, 259 (1928).

${ }^{68}$ Y. H. Xie, G. H. Gilmer, C. Roland, P. J. Silverman, S. K. Buratto, J. Y. Cheng, E. A. Fitzgerald, A. R. Kortan, S. Schuppler, M. A. Marcus, and P. H. Citrin, Phys. Rev. Lett. 73, 3006 (1994).

${ }^{69}$ D. Kandel and E. Kaxiras, Phys. Rev. Lett. 75, 2742 (1995).

${ }^{70}$ K. E. Khor and S. Das Sarma, cond-mat/0001145 (unpublished).

${ }^{71}$ A. Shklyaev, M. Shibata, and M. Ichikawa, Surf. Sci. 416, 192 (1998). 